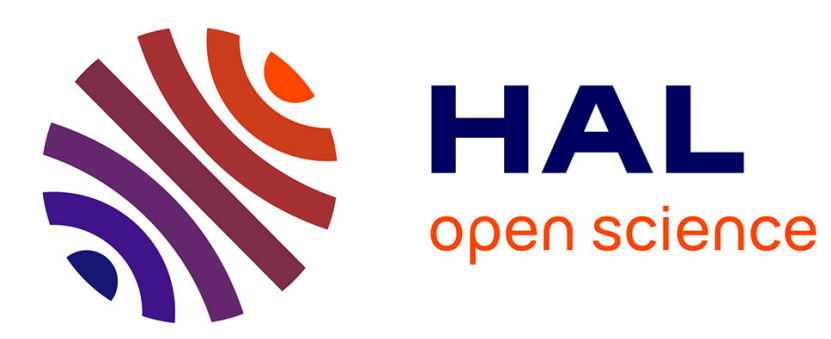

\title{
Étude des grandeurs caractéristiques de la tache cathodique d'un arc électrique
}

\author{
P. Andanson, M. Bouculat, B. Cheminat, A. Lefort, J. Roche
}

\section{To cite this version:}

P. Andanson, M. Bouculat, B. Cheminat, A. Lefort, J. Roche. Étude des grandeurs caractéristiques de la tache cathodique d'un arc électrique. Revue de Physique Appliquée, 1977, 12 (7), pp.999-1006. 10.1051/rphysap:01977001207099900 . jpa-00244283

\section{HAL Id: jpa-00244283 https://hal.science/jpa-00244283}

Submitted on 1 Jan 1977

HAL is a multi-disciplinary open access archive for the deposit and dissemination of scientific research documents, whether they are published or not. The documents may come from teaching and research institutions in France or abroad, or from public or private research centers.
L'archive ouverte pluridisciplinaire HAL, est destinée au dépôt et à la diffusion de documents scientifiques de niveau recherche, publiés ou non, émanant des établissements d'enseignement et de recherche français ou étrangers, des laboratoires publics ou privés. 


\author{
Classification \\ Physics Abstracts \\ 6.700
}

\title{
ÉTUDE DES GRANDEURS CARACTÉRISTIQUES DE LA TACHE CATHODIQUE D'UN ARC ÉLECTRIQUE $\left(^{*}\right)$
}

\author{
P. ANDANSON, M. BOUCULAT, B. CHEMINAT, A. LEFORT et J. ROCHE \\ Groupe de Physique des Matériaux \\ Université de Clermont II B. P. 4563170 Aubière, France
}

(Reçu le 9 décembre 1976, accepté le 24 mars 1977)

\begin{abstract}
Résumé. - On étudie la cathode d'un arc électrique brulant librement dans l'air pendant une demi-alternance d'un courant sinusoïdal de fréquence $50 \mathrm{~Hz}$. Nous avons déterminé pour des intensités moyennes variant entre 500 et $2000 \mathrm{~A}$ cinq grandeurs physiques caractéristiques de la tache cathodique : le rayon moyen $a$, la température $T$, le champ électrique $E$, la densité totale de courant $J$ et la fonction $s$ de la densité de courant due aux électrons. La mesure expérimentale de l'érosion de la cathode associée à un système de cinq équations a permis d'obtenir ces grandeurs pour des électrodes en cuivre et en argent. La première partie des résultats donne des valeurs moyennes, tandis que la deuxième partie montre l'évolution de la tache cathodique en fonction du temps pour la demi-alternance de courant.
\end{abstract}

\begin{abstract}
The cathode of an electric arc burning freely in air was studied during a halfwave of a sinusoidal, $50 \mathrm{~Hz}$ current. For mean intensities ranging from 500 to $2000 \mathrm{~A}$, five characteristic parameters for the cathode spot were determined : the mean radius $a$, the temperature $T$, the electric field $E$, the total current density $J$, and the fraction $s$ of the current density due to electrons. The experimental measurement of the erosion of the cathode together with five simultaneous equations enabled the parameters above to be obtained for copper and silver electrodes. The first part of the results gives mean values. The second part shows the changes occuring in the cathode spot as a function of time for the half-wave of the current.
\end{abstract}

1. Introduction. - La mesure des différentes grandeurs électriques au niveau des taches cathodiques et anodiques d'un arc électrique se révèle très difficile actuellement. Nous avons pu en effectuer la détermination d'une façon indirecte en utilisant d'une part des résultats théoriques et d'autre part des mesures sur les effets du pied d'arc à la surface de l'électrode. L'action de l'arc sur la surface des contacts se traduit par une apparition de cratères et de bosses qui ont largement été décrits par plusieurs auteurs pour différents matériaux avec des intensités et des durées d'arc très variables (voir par exemple les travaux de $\mathrm{L}$. H. Germer [1] ou de J. A. Augis et E. W. Gray [2]).

Dans notre expérience nous avons utilisé l'arc qui apparaît lors de l'ouverture d'un disjoncteur traversé par un courant électrique. Afin d'avoir des résultats expérimentaux comparables entre eux, la commande d'ouverture a été synchronisée sur le passage à zéro d'un courant électrique sinusoïdal de fréquence $50 \mathrm{~Hz}$; ce qui compte tenu du temps de réponse de l'appareillage conduit à une durée d'arc d'environ $7 \times 10^{-3} \mathrm{~s}$.

$\left(^{*}\right)$ Communication présentée au Congrès National de Physique des Plasmas, Paris 6-10 décembre 1976.
Par pesées successives des contacts nous avons déduit la valeur moyenne de l'érosion à la cathode.

A partir de cette donnée expérimentale, de la mesure du courant moyen $I$ et des hypothèses qui seront formulées en même temps que les équations théoriques, nous avons déterminé pour la tache cathodique les grandeurs suivantes :

- le rayon $a$,

- la température $T$,

- le champ électrique $E$,

- la densité totale de courant $J$,

- la fraction $s$ de la densité de courant due aux électrons.

D'autre part, nous avons effectué la mesure de l'érosion pour des durées d'arc variables, et nous avons ainsi obtenu la variation en fonction du temps de ces grandeurs pour une demi-alternance.

Nous allons maintenant donner une description succincte du dispositif expérimental.

2. Le dispositif expérimental. - La figure 1 donne le schéma du dispositif utilisé pour étudier un arc électrique sur une demi-alternance ainsi que les enre- 


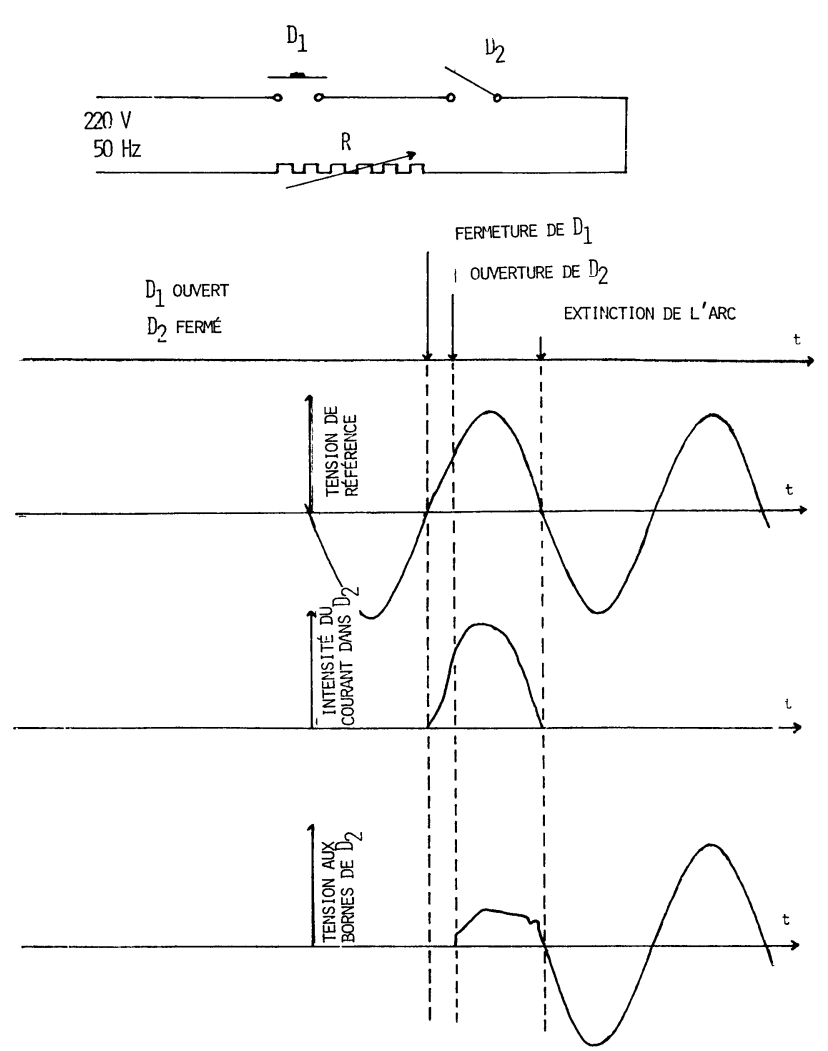

Fig. 1. - Schéma du montage permettant d'effectuer les mesures en fonction de l'intensité moyenne $I$, et les enregistrements correspondants.

[Equipment used to make measurements as a function of the average value of $I$.

gistrements de courant et de tension correspondants. La source de tension $220 \mathrm{~V}, 50 \mathrm{~Hz}$, permet d'obtenir un courant dont l'intensité peut atteindre $1000 \mathrm{~A}$ avec un déphasage $\varphi$ variable. Les résultats donnés dans cet article sont relatifs à $\varphi$ nul. L'enregistrement donné en fonction du temps $t$, sous la forme tension de référence représente la tension aux bornes de la source. La résistance $R$ permet de régler la valeur de l'intensité du courant dans le circuit. La fermeture du contacteur $\mathrm{D}_{1}$ se fait au passage à zéro de la tension de référence. L'apparition d'un courant dans le disjoncteur $\mathrm{D}_{2}$ fermé provoque l'ouverture de celui-ci. et l'apparition d'un arc électrique entre ses contacts. Cet arc s'éteint naturellement au passage à zéro de la différence de potentiel. Le graphe tension aux bornes de $\mathrm{D}_{2}$ donne la variation dans le temps de la tension d'arc suivie de la tension de rétablissement du circuit. L'enregistrement de l'intensité du courant permet de déterminer la valeur expérimentale de l'intensité moyenne $I$.

Ces graphes sont obtenus grâce à un enregistreur multivoies à développement direct sur papier sensible au rayonnement ultra-violet, sa bande passante est de $15 \mathrm{kHz}$ et il dispose d'un oscillateur base de temps avec impression sur les oscillogrammes.

$\mathrm{Ce}$ dispositif expérimental nous permet donc, par pesées des contacts du disjoncteur $D_{2}$ avant et après l'essai, de connaître l'érosion de la cathode en fonction de l'intensité moyenne $I$ avec une précision meilleure que $6 \%$.

Sur la figure 2 nous voyons la présence du contacteur $\mathrm{D}_{3}$ qui permet, comme le montrent les enregistrements de réduire la durée de vie de l'arc. Nous avons pu ainsi déterminer expérimentalement l'érosion de la cathode en fonction du temps avec une précision meilleure que $10 \%$.

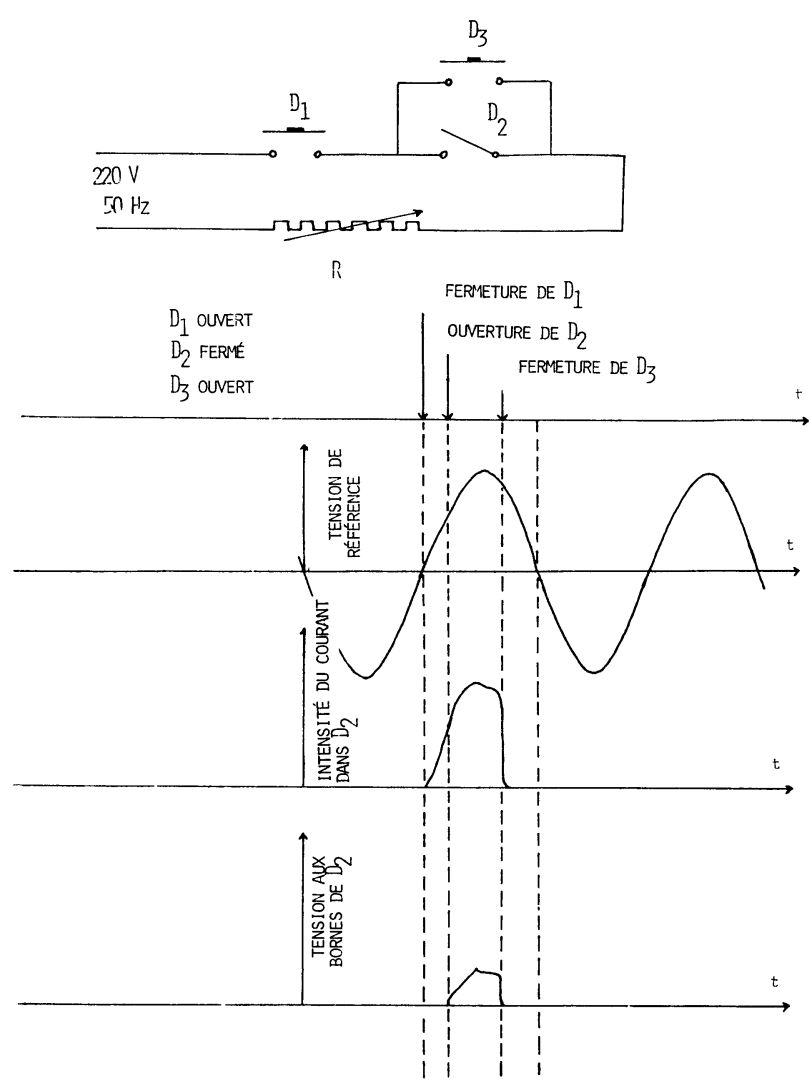

Fig. 2. - Schéma du montage permettant d'effectuer les mesures en fonction du temps $t$, et les enregistrements correspondants.

[Equipment used to make measurements as a function of time, $t$.]

3. Le système d'équations. - Avant de donner les équations relatives au problème posé nous allons formuler les hypothèses simplificatrices suivantes :

1) Les grandeurs $a, T, J, E$ et $s$ définies dans l'introduction représentent les valeurs moyennes pour un intervalle de temps donné. La réduction de cet intervalle de temps à $10^{-3}$ s permet d'obtenir l'allure de leur variation au cours de la demi-période pour laquelle nous étudions les effets de l'arc électrique au niveau de la cathode.

2) La zone étudiée s'étend devant l'électrode à surface plane sur une distance de l'ordre du libre parcours moyen des électrons. Cette distance étant faible comparée au rayon moyen a, et le champ électrique dans cette zone étant très intense et perpen- 
diculaire à la surface de l'électrode, nous supposerons somme N. P. Kozlov et V. I. Khvesyuk [3] le problème unidimensionnel.

3) Les travaux de P. R. Emtage [4] montrent que la tache cathodique est formée d'une multiplicité de taches identiques qui d'après R. B. Nagaibekov [5] ont une durée de vie moyenne comprise entre $10^{-6}$ et $10^{-5} \mathrm{~s}$. Nous considérons qu'au niveau des échanges à la surface de la cathode, les effets et les surfaces de toutes ces taches cathodiques s'ajoutent de façon à être comparables à ceux d'une tache circulaire unique se déplaçant très peu et de rayon $a$.

4) Nous supposons que l'érosion des contacts a lieu uniquement par évaporation. Cette hypothèse est de moins en moins valable lorsque l'intensité $I$ dépasse une certaine valeur, surtout pour les électrodes en cuivre. Nous confirmons cette difficulté par un examen expérimental au niveau du phénomène et nous verrons en conclusion comment cette hypothèse peut conduire à une imprécision sur nos résultats.

5) Nous supposons que tous les ions atteignant la cathode sont neutralisés sur celle-ci et ne rebondissent pas. Ceci nous conduit d'après I. N. Ostretsov et al. [6] à des valeurs du champ électrique trop élevées, mais d'après ces auteurs la correction est faible et devient négligeable pour des champs électriques de l'ordre de ceux que nous avons obtenus, vu notre précision expérimentale.

6) Pour le bilan de puissance, nous supposons que les échanges d'énergie au niveau de la tache cathodique ont lieu instantanément en comparaison du temps d'arc, même lorsque celui-ci se réduit à $10^{-3} \mathrm{~s}$.

Dès l'apparition de l'arc électrique, il se produit une évaporation métallique à la cathode ; la présence d'un courant d'électrons rapides provoque l'ionisation partielle de la vapeur métallique. Il y a une accumulation de charges entre la tache cathodique et la colonne d'arc, ce qui crée une charge d'espace importante dont il faut tenir compte pour le calcul du champ électrique. Ce calcul effectué par Mackeown [7] conduit à l'équation suivante :

$E=\left\{\frac{4}{\varepsilon_{0}} J\left((1-s)\left(\frac{M}{2 e}\right)^{1 / 2}-s\left(\frac{m}{2 e}\right)^{1 / 2}\right) V_{c}^{1 / 2}\right\}^{1 / 2}$

où $\varepsilon_{0}$ représente la permittivité du vide, $M$ la masse atomique des ions de la charge d'espace, $m$ et $e$ respectivement la masse et la charge de l'électron, et $V_{\mathrm{c}}$ la chute cathodique. Les valeurs expérimentales de $V_{c}$ sont empruntées à V. E. Grakov [8].

La sortie des électrons au niveau de la cathode est fonction de la température et du champ électrique à la surface de celle-ci. La plupart des auteurs distinguent essentiellement trois cas :

- émission thermoélectronique lorsque la température est élevée ;

- émission par effet de champ lorsque celui-ci prend des valeurs supérieures à $10^{9} \mathrm{~V} . \mathrm{m}^{-1}$;

- émission par effet de champ et par effet thermoélectronique lorsque les valeurs du champ et de la température sont simultanément élevées.

Nous avons utilisé pour calculer la densité $J_{\mathrm{e}} \mathrm{du}$ courant électronique émis la relation de Murphy et Good [9] qui est valable pour les trois types d'émission :

$$
J_{\mathrm{e}}=\int_{-W_{\mathbf{A}}}^{\infty} D(E, W) N(T, W) \mathrm{d} W .
$$

$D(E, W)$ est la probabilité d'émission d'un électron d'énergie $W$ du métal soumis à un champ électronique $E ; N(T, W) \mathrm{d} W$ est le nombre d'électrons frappant la barrière de potentiel par unité de surface et par seconde et ayant une énergie comprise entre $W$ et $W+\mathrm{d} W$, et $W$ est le travail d'extraction du métal.

En remplaçant $D(E, W)$ et $N(T, W)$ par les valeurs données par ces auteurs, nous sommes conduits à l'espression :

$$
\begin{array}{r}
J_{\mathrm{e}}=\frac{4 \pi m k T \mathrm{e}}{h^{3}} \int_{-W_{\mathrm{a}}}^{W_{l}} \exp \left(-\frac{W-\xi}{k T}-\frac{8 \pi \sqrt{2} m^{1 / 2} e^{5 / 4} v(y)}{3\left(4 \pi \varepsilon_{0}\right)^{3 / 4} h E^{1 / 4} y^{3 / 2}}\right) \cdot \mathrm{d} W+ \\
\quad+\frac{4 \pi m k T \mathrm{e}}{h^{3}} \int_{W_{l}}^{\infty} \ln \left(1+\exp \left[-\frac{W-\xi}{k T}\right]\right) \cdot \mathrm{d} W
\end{array}
$$

où $h$ est la constante de Planck, $k$ la constante de Boltzmann, $W$ l'énergie de l'électron, $\xi$ le niveau de Fermi ; $W_{l}$ et $y$ valent :

$$
W_{l}=-\frac{1}{2}\left(2 e^{3} E\right)^{1 / 2} ; \quad y=\frac{\left(e^{3} E\right)^{1 / 2}}{|W|} .
$$

La fonction $v(y)$ se calcule à partir d'intégrales elliptiques que l'on peut trouver dans l'article de Murphy et Good.
La définition de la fraction électronique s donne la relation :

$$
J_{\mathrm{e}}=s J .
$$

En supposant la tache cathodique circulaire et de rayon $a$, nous avons entre $I$ et $J$ la relation :

$$
I=\pi a^{2} J \text {. }
$$

Nous faisons l'hypothèse que la perte de masse à la cathode se fait essentiellement par évaporation du 
métal au niveau du pied d'arc. A partir des mesures que nous avons effectuées, nous déterminons la perte massique par unité de temps $\Delta m$. Les travaux de S. Dushman [10] sur la puissance nécessaire à l'évaporation des métaux dans le vide permettent de relier $\Delta m$ à la température $T$ de la tache cathodique par la relation :

$$
\Delta m=C \cdot \pi \cdot a^{2} \cdot 10^{\alpha(T)}
$$

avec

$$
\alpha(T)=A-\frac{1}{2} \log _{10} T-\frac{B}{T} .
$$

$C$ est une constante valant $1 \mathrm{~kg} \cdot \mathrm{m}^{-2} \cdot \mathrm{s}^{-1} ; A$ et $B$ sont deux constantes qui dépendent de la nature du matériau évaporé. Il faut remarquer que cette relation n'est valable que pour le vide, c'est-à-dire ici la cathode en présence de sa propre vapeur métallique. L'arc se développe dans un milieu qui, au départ, du fait de l'ouverture des contacts est à une pression inférieure à la pression atmosphérique.

La formule de Lee et A. Greenwood [11] donne la pression maximale au niveau de la tache cathodique :

$$
P_{\mathrm{m}}=K^{\prime} . I . J+P_{\mathrm{a}} .
$$

$K^{\prime}$ vaut $10^{-7} \mathrm{~Pa} \cdot \mathrm{A}^{-2} \cdot \mathrm{m}^{2}$ si la pression maximale $P_{\mathrm{m}}$ et la pression ambiante $P_{\mathrm{a}}$ sont exprimées en Pascal

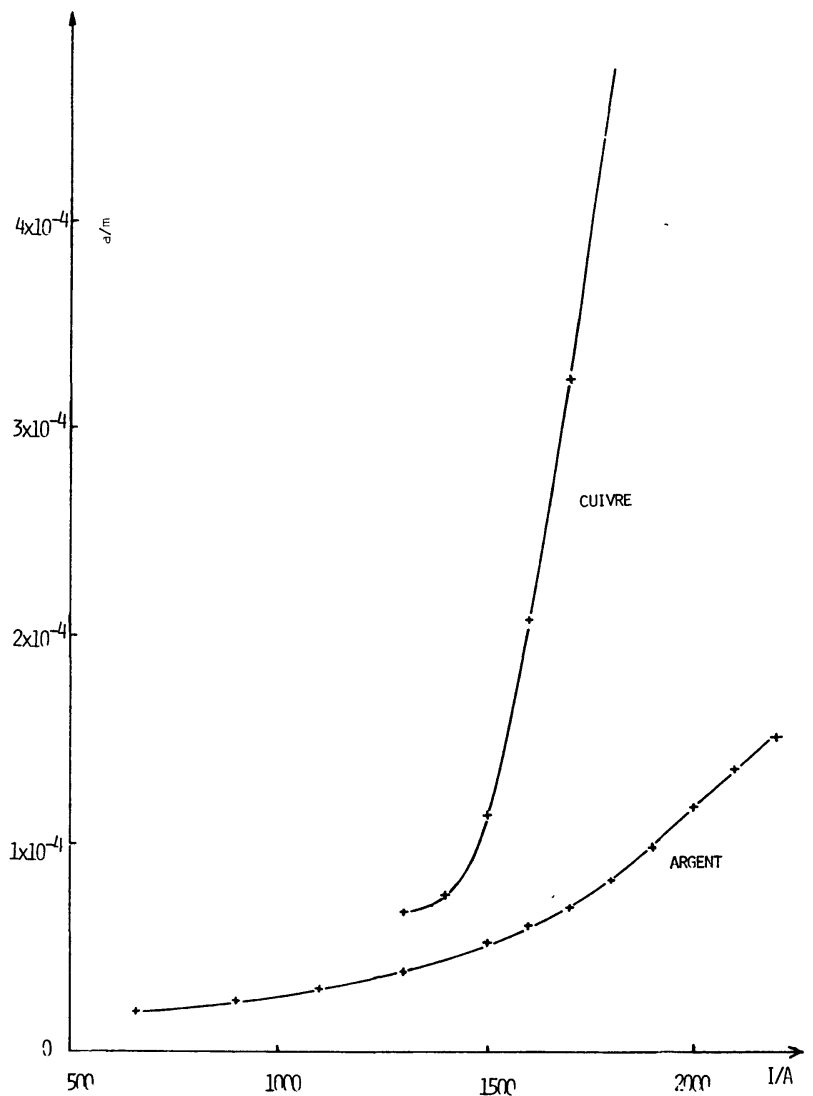

Fig. 3. - Variation du rayon $a$ de la tache cathodique en fonction du courant moyen $I$. (Incertitude absolue inférieure à $0,6 \times 10^{-5} \mathrm{~m}$.)

[Variation of the radius $a$ of the cathode spot as a function of the mean current $I$.]
$I$ et $J$ ayant respectivement pour unité des $A$ et des $A \cdot \mathrm{m}^{-2}$. Ceci nous conduit à des pressions partielles pour les vapeurs métalliques de l'ordre de $10^{7}$ Pascal ce qui justifie l'utilisation de la relation (6).

Le bilan énergétique au niveau de la tache cathodique conduit à l'égalité :

$$
P_{\mathrm{i}}=P_{\mathrm{v}}+P_{\mathrm{c}}+P_{\mathrm{r}}+P_{\mathrm{e}} .
$$

La puissance fournie $P_{\mathbf{i}}$ est égale à la somme des puissances dissipées : par évaporation métallique $\left(P_{\mathrm{v}}\right)$, par conduction $\left(P_{\mathrm{c}}\right)$, par rayonnement $\left(P_{\mathrm{r}}\right)$ et par évaporation des électrons $\left(P_{\mathrm{e}}\right)$. La puissance $P_{\mathrm{i}}$ résultant du bombardement de la cathode par les ions métalliques s'exprime en fonction de $J, s$ et $a$ :

$$
P_{\mathrm{i}}=(1-s) J\left(V_{\mathrm{i}}+V_{\mathrm{c}}-V_{\mathrm{a}}\right) \cdot \pi \cdot a^{2} .
$$

$V_{\mathrm{i}}$ est le potentiel d'ionisation et $V_{\mathrm{a}}$ le potentiel correspondant au travail d'extraction $W_{\mathrm{a}}$ pour le métal constituant la cathode. Examinons maintenant les termes du deuxième membre de la relation (8).

La puissance dissipée par l'évaporation métallique s'obtient directement à partir de la relation (6) :

$$
P_{\mathrm{v}}=D . \Delta m \text {. }
$$

$D$ représente le travail nécessaire pour évaporer l'unité de masse du métal constituant la cathode.

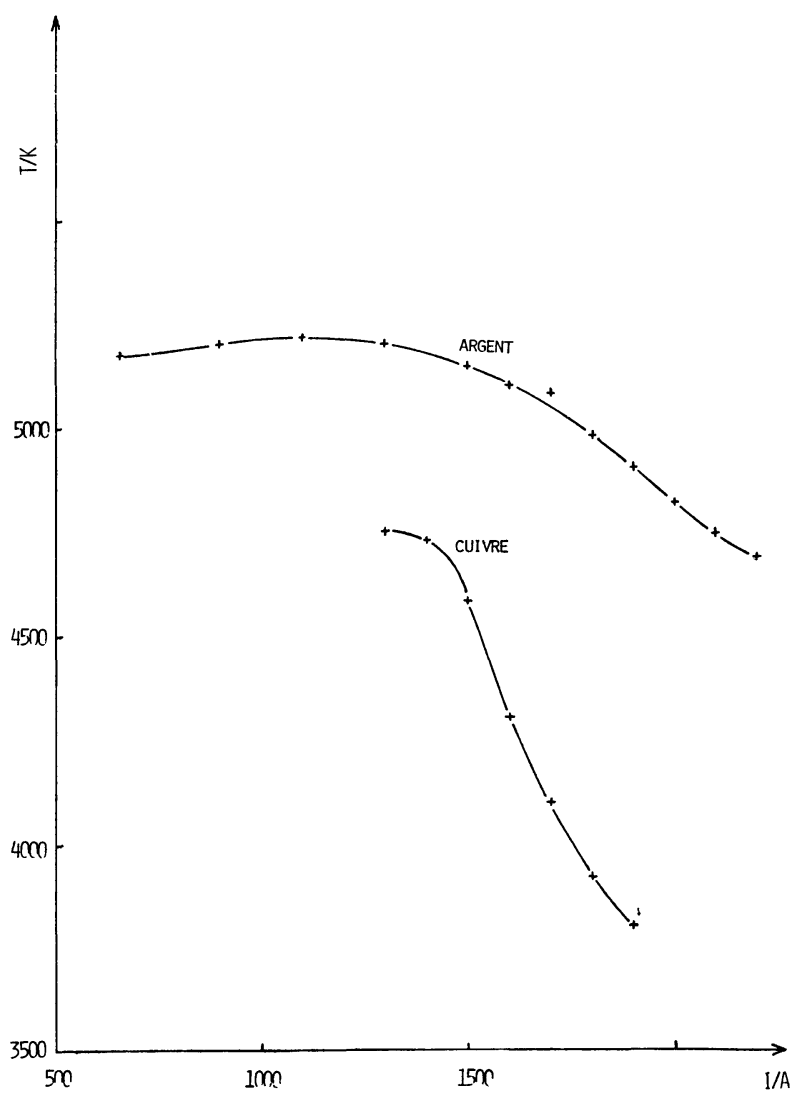

Fig. 4. - Variation de la température $T$ de la tache cathodique en fonction du courant moyen $I$. (Incertitude absolue inférieure à $50 \mathrm{~K}$.)

[Variation of the temperature $T$ of the cathode spot as a function of the mean current $I$.] 


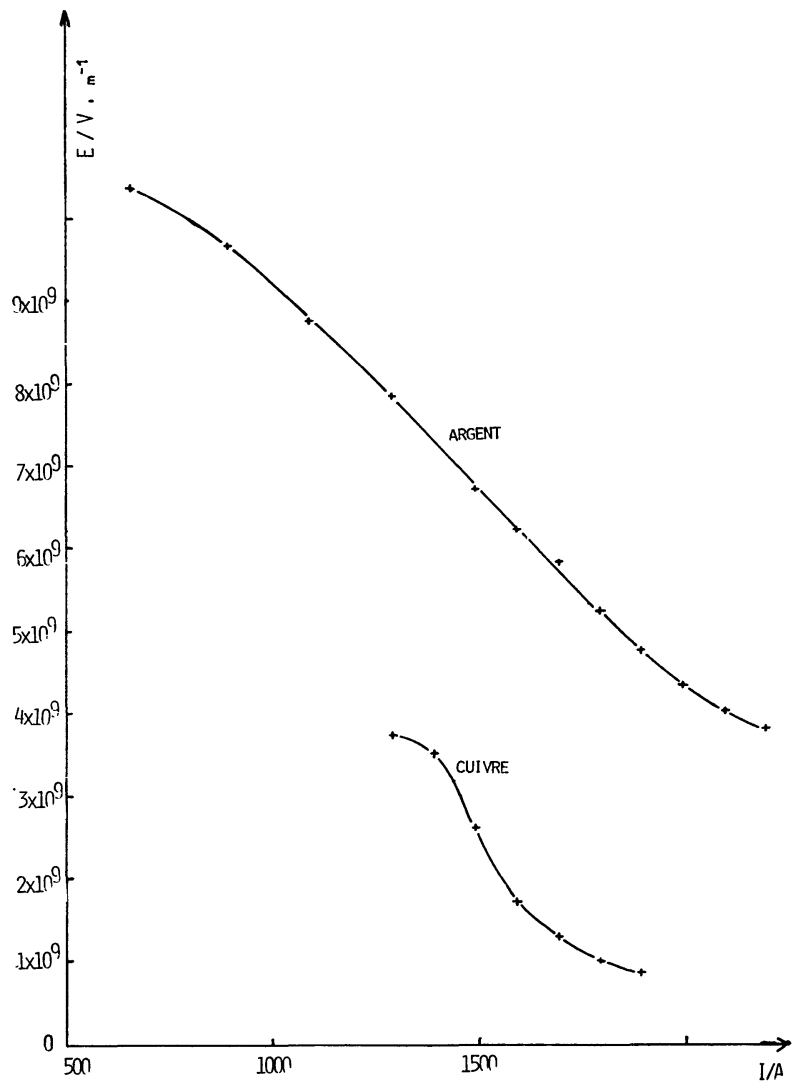

Fig. 5. - Variation du champ électrique $E$ de la tache cathodique en fonction du courant moyen $I$. (Incertitude absolue inférieure à $0,5 \times 10^{9} \mathrm{~V} \cdot \mathrm{m}^{-1}$.)

[Variation of the electric field $E$ at the cathode spot as a function of the mean current $I$.]

La résolution de l'équation de la conduction de la chaleur, en supposant que le régime permanent est très rapidement atteint donne avec une bonne approximation la puissance $P_{\mathrm{c}}$ dissipée par conduction :

$$
P_{\mathrm{c}}=\left(T-T_{0}\right) K \cdot \pi^{3 / 2} \cdot a
$$

où $K$ représente la conductivité thermique de la cathode et $T_{0}$ la température ambiante.

Un calcul rapide montre que la puissance perdue par rayonnement $P_{\mathrm{r}}$ est très faible et négligeable devant les autres termes, nous la considérerons comme nulle pour la suite.

D'après Lee [12], l'effet de refroidissement des électrons quittant la cathode vaut en moyenne $W_{\mathrm{r}}=2 \mathrm{eV}$ par électron, ce qui conduit pour $P_{\mathrm{e}}$ à la valeur :

$$
P_{\mathrm{e}}=W_{\mathrm{r}} \pi a^{2} s J
$$

Nous avons donc obtenu un système de cinq équations constitué par les relations (1), (4), (5), (6) et (8) reliant les cinq grandeurs $a, T, E, J$ et $s$.

4. Les résultats. -4.1 VARIATION DES GRANDEURS CARACTÉRISTIQUES DE LA CATHODE AVEC L'INTENSITÉ MOYENNE. - La résolution d'un tel système n'a pu se

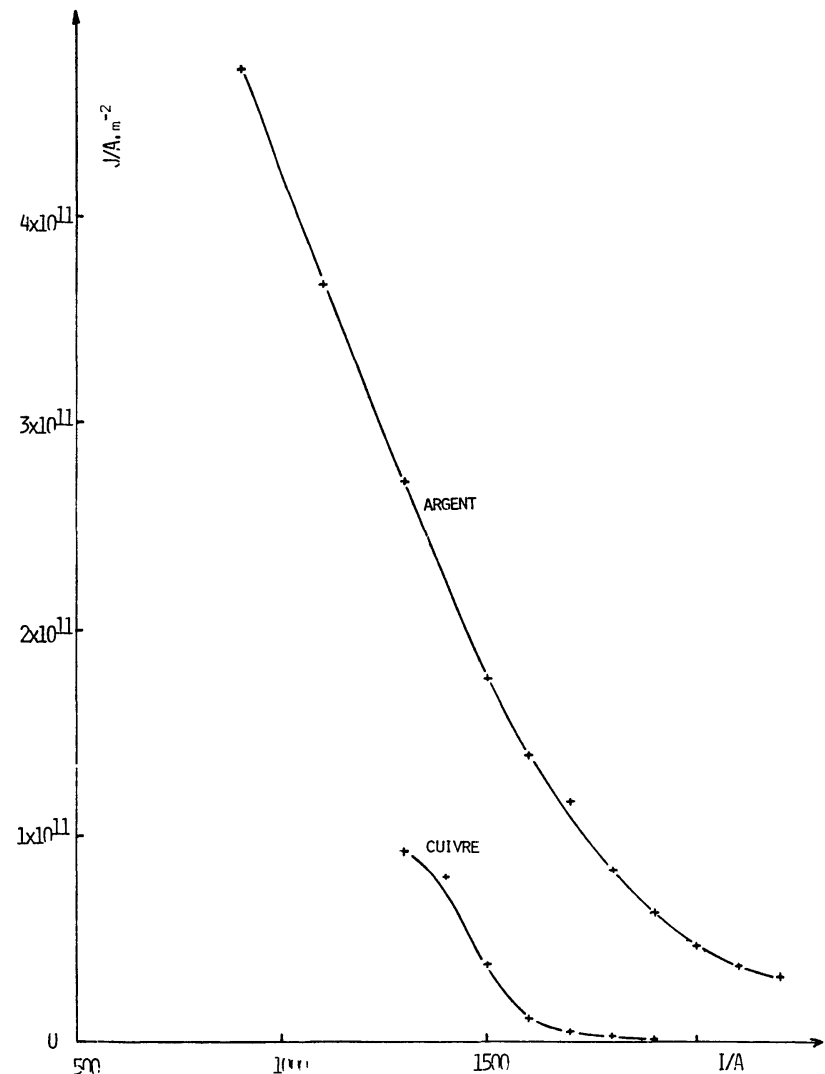

Fig. 6. - Variation de la densité de courant $J$ de la tache cathodique en fonction du courant moyen $I$. (Incertitude absolue inférieur à $3 \times 10^{10} \mathrm{~A} . \mathrm{m}^{-2}$.)

[Variation of the current density $J$ of the cathode spot as a function of the mean current $I$.]

faire qu'à l'aide d'un calculateur numérique. Le calcul a été effectué de la façon suivante : pour chaque couple des valeurs $\Delta m$ et $I$ qui constituent les données expérimentales nous avons calculé $J$ de deux façons différentes en prenant la température $T$ comme paramètre, lorsque les deux calculs conduisent à la même valeur de $J$ le système est résolu.

Nous donnons dans les figures 3, 4, 5, 6 et 7 les résultats obtenus pour des électrodes en cuivre et en argent. La comparaison entre les deux métaux montre tout d'abord que les variations des grandeurs étudiées se font dans le même sens pour les deux cas. D'autre part nous remarquons pour le cuivre une absence de résultats pour des intensités moyennes inférieures à $1300 \mathrm{~A}$, ceci est dû au fait que pour les basses intensités les valeurs de $\Delta m$ sont entachées d'une erreur relative trop importante pour permettre un calcul raisonnable.

Le calcul d'erreur relatif à ces résultats ainsi qu'à ceux du paragraphe suivant a été obtenu en effectuant la résolution du système pour les deux extrémités des barres d'erreur de la courbe de la perte de masse. Ceci est possible car l'erreur relative sur l'intensité moyenne $I$ est négligeable devant celle sur la perte de masse. Les incertitudes obtenues sont données avec les légendes des différentes courbes. 


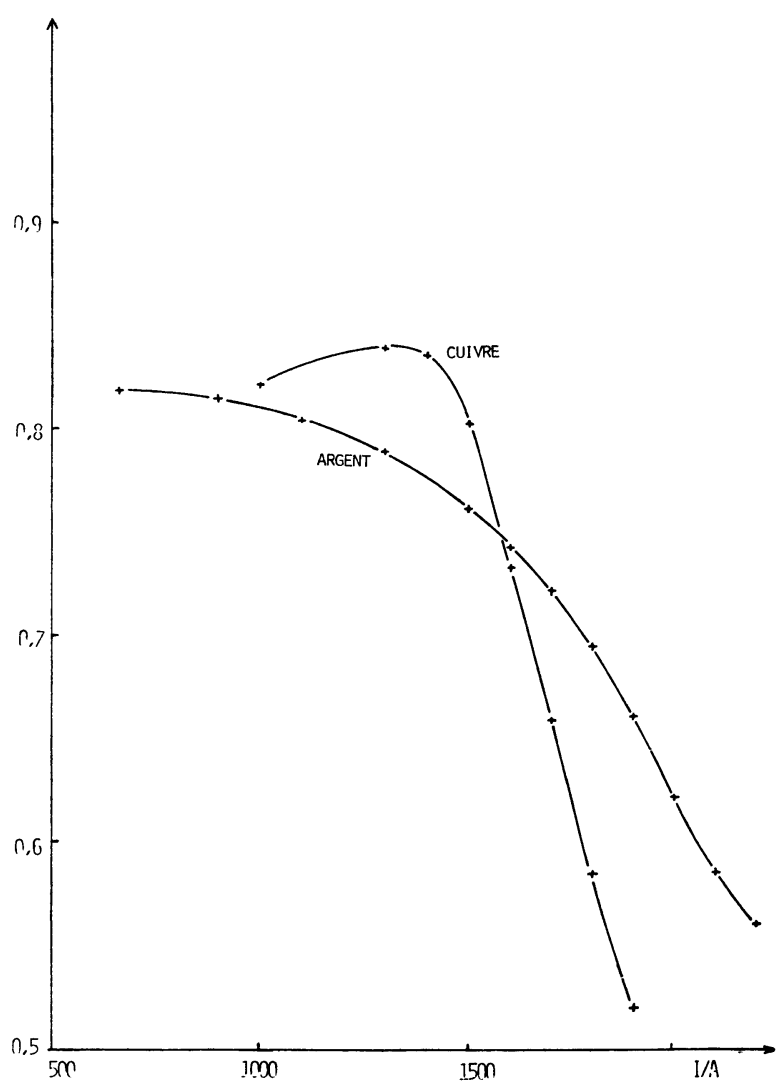

Fig. 7. - Variation de la fraction électronique $s$ au niveau de la tache cathodique en fonction du courant moyen $I$. (Incertitude absolue inférieure à 0,03 .)

[Variation of the electronic fraction $s$ at the cathode spot as a function of the mean current $I$.]

\subsection{VARIATION DES GRANDEURS CARACTÉRISTIQUES} DE LA CATHODE AVEC LE TEMPS. - Dans la série d'expériences précédemment décrites l'arc électrique s'éteint naturellement au passage à zéro du courant sinusoïdal si celui-ci est en phase avec la tension.

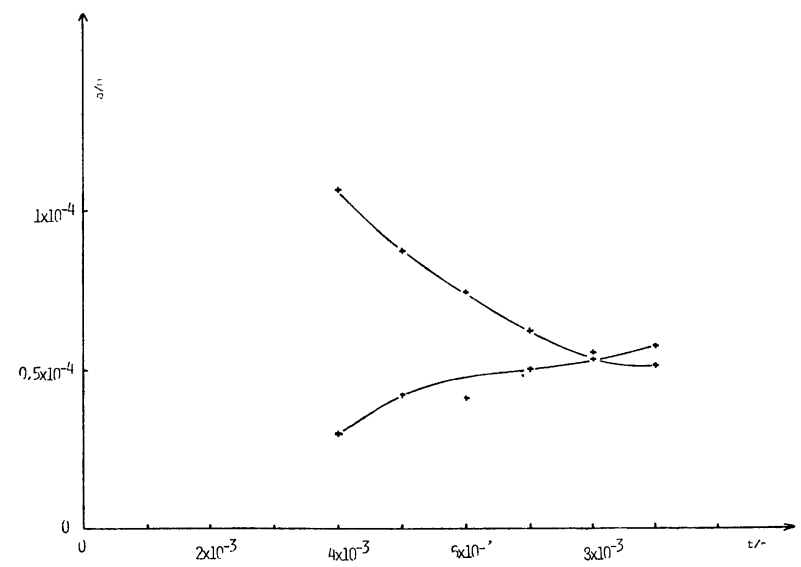

Fig. 8. - Variation du rayon $a$ de la tache cathodique en fonction du temps pour une cathode en cuivre (courant efficace d'arc de $2200 \mathrm{~A}$ ) et pour une cathode en argent (courant efficace d'arc de $1500 \mathrm{~A}$ ). (Incertitude absolue inférieure à $1 \times 10^{-5} \mathrm{~m}$.) [Variation of the radius $a$ of the cathode spot as a function of time for a copper cathode (effective arc current : $2200 \mathrm{~A}$ ) and for a silver cathode (effective arc current : $1500 \mathrm{~A}$ ).]
Nous avons pu, grâce au système expérimental décrit dans le paragraphe 2, éteindre l'arc électrique à un instant choisi. Ceci nous a permis de connaître la perte massique de la cathode en fonction du temps pour une intensité efficace donnée. Nous avons pu ainsi déterminer les variations des grandeurs caractéristiques à la cathode en fonction du temps, pour une demialternance. Nous donnons les résultats dans les figures $8,9,10,11$ et 12 pour des contacts en argent traversés par un courant d'intensité efficace de $1500 \mathrm{~A}$ et pour

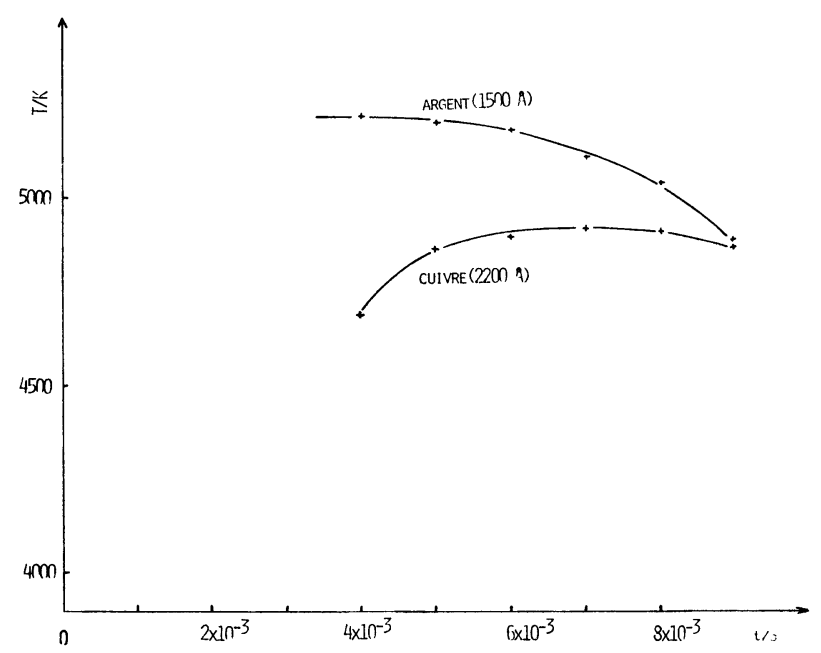

Fig. 9. - Variation de la température $T$ de la tache cathodique en fonction du temps pour une cathode en cuivre (courant efficace d'arc de $2200 \mathrm{~A}$ ) et pour une cathode en argent (courant efficace d'arc de $1500 \mathrm{~A}$ ). (Incertitude absolue inférieure à $100 \mathrm{~K}$.

[Variation of the temperature $T$ of the cathode spot as a function of time for a copper cathode (efficace arc current : $2200 \mathrm{~A}$ ) and for a silver cathode (effective arc current : $1500 \mathrm{~A}$ ).]

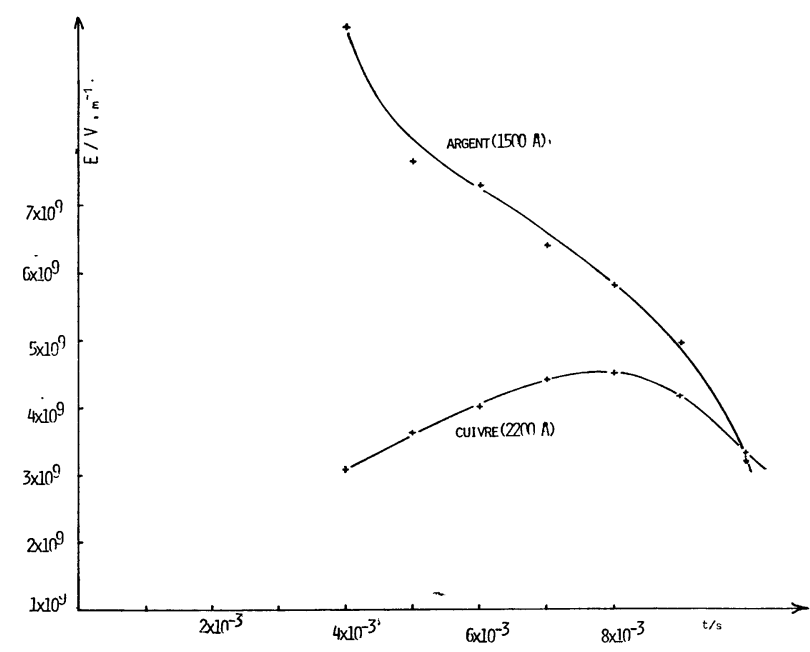

Fig. 10. - Variation du champ électrique $E$ de la tache cathodique en fonction du temps pour une cathode en cuivre (courant efficace d'arc de $2200 \mathrm{~A}$ ) et pour une cathode en argent (courant efficace d'arc de $1500 \mathrm{~A}$ ). (Incertitude absolue inférieure à $0,6 \times 10^{9} \mathrm{~V} . \mathrm{m}^{-1}$.)

[Variation of the electric field $E$ at the cathode spot as a function of time for a copper cathode (effective arc current : $2200 \mathrm{~A}$ ) and for a silver cathode (effective arc current : $1500 \mathrm{~A}$.] 


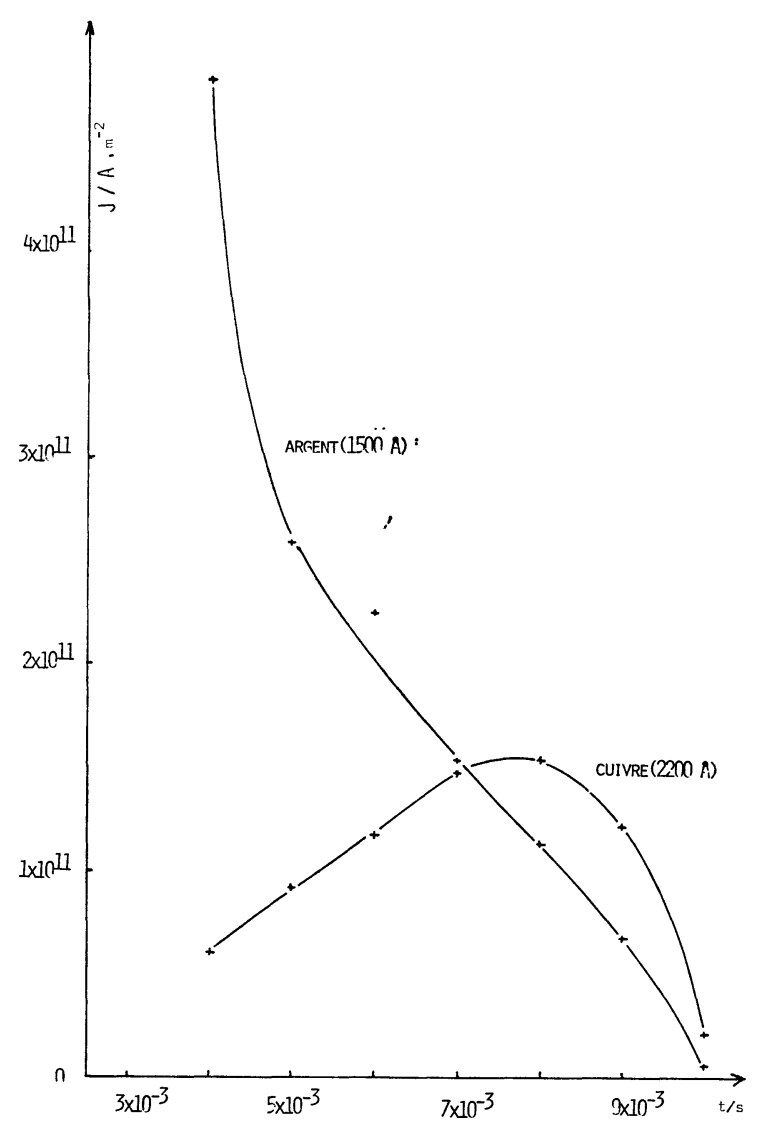

Fig. 11. - Variation de la densité de courant $J$ de la tache cathodique en fonction du temps pour une cathode en cuivre (courant efficace d'arc de $2200 \mathrm{~A}$ ) et pour une cathode en argent (courant efficace d'arc de $1500 \mathrm{~A}$ ). (Incertitude absolue inférieure à $4 \times 10^{10} \mathrm{~A} \cdot \mathrm{m}^{-2}$.)

[Variation of the current density $J$ of the cathode sport as a function of time for a copper cathode (effective arc current : $2200 \mathrm{~A}$ ) and for a silver cathode (effective arc current :1500 A).]

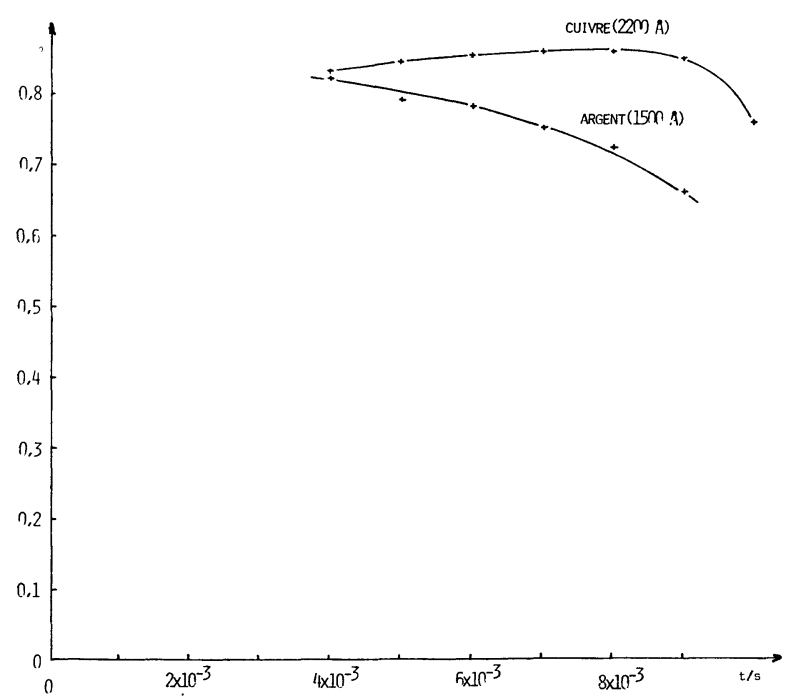

Fig. 12. - Variation de la fraction électronique $s$ en fonction du temps pour une cathode en cuivre (courant efficace d'arc de $2200 \mathrm{~A}$ ) et pour une cathode en argent (courant efficace d'arc de $1500 \mathrm{~A}$ ). (Incertitude absolue inférieure à 0,04.)

[Variation of the electronic fraction $s$ as a function of time for a copper cathode (effective arc current : $2200 \mathrm{~A}$ ) and for a silver cathode (effective arc current : $1500 \mathrm{~A}$ ).] des contacts en cuivre traversés par un courant d'intensité efficace de $2200 \mathrm{~A}$.

Les valeurs obtenues concernent l'intervalle de temps $4 \times 10^{-3} \mathrm{~s}$ à $10 \times 10^{-3} \mathrm{~s}$, si on fixe pour instant zéro le passage par zéro du courant. Le temps de réponse de la mécanique du disjoncteur étant de $3 \times 10^{-3} \mathrm{~s}$, nous voyons que nous connaissons les paramètres de la tache cathodique à partir d'un instant qui se situe $10^{-3} \mathrm{~s}$ après l'apparition de l'arc.

Il est remarquable de voir que la température très élevée de la tache cathodique est atteinte pour une durée d'arc inférieure à $10^{-3} \mathrm{~s}$; et que cette température varie ensuite relativement peu au cours du temps. Nous pouvons faire une conclusion semblable pour la fraction électronique $s$ qui varie autour de la valeur 0,8 pour le cuivre et autour de la valeur 0,75 pour l'argent.

5. Conclusion. - Nous allons maintenant comparer nos résultats à ceux proposés par différents auteurs.

L'examen des courbes donnant la densité de courant $J$ montre que nos valeurs sont en accord avec celles de de C. C. Sanger et P. E. Secker [13] qui ont examiné au microscope électronique les traces laissées par la tache cathodique. Ils concluent en donnant pour le cas d'une électrode en cuivre une valeur moyenne de $J$ de $10^{11} \mathrm{~A} \cdot \mathrm{m}^{-2}$. De même pour la fraction électronique nous confirmons les prévisions théoriques de Erk [14] qui la situe dans l'intervalle 0,5-0,8. Pour le champ électrique nous retrouvons la zone $10^{9}-10^{10} \mathrm{~V} \cdot \mathrm{m}^{-1}$ pour laquelle Lee [15] présente ses calculs sur la théorie de l'émission des électrons à la cathode d'un arc électrique.

Les résultats expérimentaux concernant la température de la tache cathodique pour les deux métaux étudiés ici et pour des intensités aussi importantes sont assez rares, aussi nous ne pourrons qu'effectuer une comparaison qualitative. Tout d'abord nous remarquons, en regardant la figure 4, que la température de la tache cathodique face à une électrode en cuivre semble décroître assez rapidement si nous la comparons à celle obtenue, pour une électrode en argent. Ceci est dû au fait que dans le cas d'une électrode en cuivre pour des intensités élevées il se superpose à l'érosion par évaporation une érosion constituée par des projections de particules qui fausse les résultats. Une extrapolation des courbes relatives au cuivre doit donc se faire selon le sens des variations de la courbe relative à l'argent qui ne présente par ce phénomène de projection. Dans ce cas nous voyons que pour des intensités plus faibles que celles données sur la figure 4, nous aurons des températures de la tache cathodique voisines de $4000 \mathrm{~K}$. Cette valeur extrapolée n'est bien sûr qu'indicative et confirme les travaux de J. C. Scherman et al. [16] qui montre que l'existence du pied d'arc nécessite une température de $4000 \mathrm{~K}$ pour une intensité du courant de $100 \mathrm{~A}$ ainsi que les travaux de T. Utsumi [17] qui trouve $2570 \mathrm{~K}$ pour des courants inférieurs à $50 \mathrm{~A}$. D'autre 
part, le fait que le rapport des énergies de sublimation du cuivre et de l'argent soit voisin de 2 et que le rapport de l'érosion entre les électrodes en argent et en cuivre soit inférieur à 2 pour les valeurs des intensités utilisées, montre que la tache cathodique cède plus d'énergie dans le cas d'une cathode en cuivre ce qui conduit à une température plus basse comme le montre la figure 4. Ce phénomène d'échange thermique plus important dans le cas du cuivre est accentué par la différence des rayons de la tache cathodique donnés figure 3.

D'autre part, le rayon de la tache cathodique croît avec l'intensité comme on pouvait le prévoir, mais il varie beaucoup plus rapidement pour le cuivre que pour l'argent ce que confirme l'examen des contacts.

Pour terminer, nous dirons que l'arc électrique crée au niveau de la tache cathodique une liaison entre un plasma dont la température atteint plusieurs milliers de Kelvin, et une électrode métallique qui, au départ est à la température ambiante. L'étude de cette zone nous montre que la tenue des contacts est liée à leurs propriétés physiques et conduit à l'utilisation, par les constructeurs d'appareils de coupure, d'alliages spéciaux à base d'argent qui assure une faible résistance de contact auquel est ajouté un ou plusieurs composés qui assurent une meilleure solidité.

\section{Bibliographie}

[1] Germer, L. H., J. Appl. Phys. 29 (1958) 1067.

[2] Augis, J. A. et Gray, E. W., J. Appl. Phys. 42 (1971) 3367.

[3] Koslov, N. P. et Khvesyuk, V. I., Sov. Phys. Tech. Phys. 16 (1972) 1691.

[4] Emtage, P. R., J. Appl. Phys. 46 (1975) 3809.

[5] Nagaibekov, R. B., Sov. Phys. Tech. Phys. 18 (1974) 1259.

[6] Ostretsov, I. N., Petrosov, V. A., Porotnikov, A. A. et RadNovich, B. B., High Temp. 12 (1974) 1143.

[7] Mackeown, S. S., Phys. Rev. 34 (1929) 611.

[8] Grakov, V. E., Zh. Tekh. Fiz. 37 (1967) 396.

[9] Murphy, E. L. et Good, T. H., Phys. Rev. 102 (1956) 1464.
[10] Dushman, S., Vacuum Technique (John Wiley and Sons, Inc. New-York) 1949742

[11] Lee, T. H. et Greenwood, A., J. Appl. Phys. 32 (1961) 916.

[12] Lee, T. H., J. Appl. Phys. 31 (1960) 924.

[13] Sanger, C. C. et SeCKer, P. E., J. Phys. D. 4 (1971) 1940.

[14] ERK, A., SChMelzLe, M., Grundlagen der Schaltgerätetechnik (Springer Verlag, Berlin. Heidelberg. New-York) 197460.

[15] Lee, T. H., J. Appl. Phys. 30 (1959) 166.

[16] Sherman, J. C., Webster, R., Jenkins, J. E. et Holm es, R. J. Phys. D. 8 (1975) 696.

[17] Utsumi, T., Appl. Phys. Lett. 18 (1971) 218. 